\title{
Friction Behavior of a Wet Clutch Subjected to Accelerated Degradation
}

\author{
Agusmian Partogi Ompusunggu, ${ }^{1}$ Thierry Janssens, ${ }^{2}$ and Paul Sas ${ }^{2}$ \\ ${ }^{1}$ Flanders' Mechatronics Technology Centre (FMTC), Celestijnenlaan 300D, 3001 Heverlee, Belgium \\ ${ }^{2}$ Division PMA, Department of Mechanical Engineering, Katholieke Universiteit Leuven (KU Leuven), Celestijnenlaan 300B, \\ 3001 Heverlee, Belgium
}

Correspondence should be addressed to Agusmian Partogi Ompusunggu; agusmian.ompusunggu@gmail.com

Received 19 November 2012; Accepted 10 December 2012

Academic Editors: D. Das, M. Dienwiebel, and J. Wang

Copyright (C) 2013 Agusmian Partogi Ompusunggu et al. This is an open access article distributed under the Creative Commons Attribution License, which permits unrestricted use, distribution, and reproduction in any medium, provided the original work is properly cited.

\begin{abstract}
This study aims at experimentally investigating the sliding friction characteristics of a wet clutch during its lifetime. More precisely, the objective is to understand how the Stribeck and the frictional lag (i.e, sliding hysteresis) parameters evolve as the clutch degradation progresses. For this purpose, a novel test procedure has been proposed and a set of experiments has been carried out on a fully assembled (commercial) clutch using a modified SAE\#2 test setup. Furthermore, a systematic methodology for the Stribeck and the frictional lag parameters identification is developed. Regardless of the applied pressure, it appears that the first three identified Stribeck parameters tend to decrease with the progression of the degradation, while the last parameter tends to increase. In regard to the frictional lag parameter, the trend shows pressure dependency.
\end{abstract}

\section{Introduction}

Adhesive wear and thermal degradation are the main aging sources of clutch friction materials, which are unavoidably present when clutches are in operation. The dominance of these aging sources is determined by many factors, such as the used friction material, oil, and operational condition, Regarding the sliding friction, the characteristics can be classified into two categories, namely, (1) the stationary friction characteristic and (2) the dynamic friction characteristic $[1,2]$. As discussed in the literature, the stationary friction characteristic is typified by the Stribeck curve while the dynamic one is typified by the frictional lag. To the authors' knowledge, how the Stribeck curve and the frictional lag evolve with the progression of the friction material degradation is not fully understood yet. No articles have been found in the open literature that address this issue.

This study aims at experimentally investigating the typical sliding friction characteristics of a wet friction clutch during its lifetime. More precisely, the objective of the study is to understand how the Stribeck and the frictional lag (i.e., sliding hysteresis) parameters evolve as the degradation progresses. A profound understanding of the evolution of the Stribeck parameters and the sliding hysteresis loop during clutch lifetime may allow to model the evolution of the clutch friction characteristics during the lifetime. This model can then be integrated to a clutch model such that simulations of the dynamic engagement behavior of the clutch with the progression of the friction material degradation is possible. Eventually, the gained knowledge can lead to the derivation of physical features, which are useful for developing a clutch monitoring and prognostic system.

For this purpose, a novel test procedure has been proposed and applied on a fully assembled (commercial) clutch tested on an SAE\#2 test setup. The accelerated life test (ALT) concept is utilized to accelerate the progression of the clutch friction material degradation, wherein two additional tests, namely, (i) stationary Stribeck test and (ii) dynamic Stribeck test, are performed between predefined number of duty cycles. This way, important sliding friction characteristics 
of clutches can be systematically evaluated. The used clutch consists of a predefined number of commercial friction discs and separator discs that are lubricated with a commercial ATF.

The remainder of this paper is organized as follows. The experimental aspects comprising the test setup used in the study and the experimental procedures are presented and discussed in Section 2. The results obtained from the experiments are then presented and discussed in Section 3, where the parameter identification technique and the identification results are also discussed in this section. Finally, some important conclusions drawn from the study are summarized in Section 4.

\section{Experimental Aspects}

This section begins with some details of the SAE\#2 test setup used in the study. An overview concerning the used friction disc, the separator disc, and the ATF is also given. Finally, the experimental procedures carried out in the study are then discussed in the last part of this section.

2.1. SAE\#2 Test Setup. According to the standard of Society of Automotive Engineer (SAE) (i.e., SAE J2489) [3], an SAE\#2 test setup is used to evaluate the friction characteristics of automatic transmission clutches with automotive transmission fluids (ATFs). It can also be used to conduct durability tests on wet friction clutch systems and to evaluate the performance variation as a function of the number of duty cycles. Normally, a typical SAE\#2 test setup is equipped with a flywheel driven by an electric motor and the kinetic energy of this wheel is dissipated in a tested clutch [4]. As the main objective of the current study is to perform the stationary and dynamic Stribeck tests that will be discussed in the forthcoming paragraphs, the presence of a flywheel in an SAE\#2 might lead to difficulties in conducting both tests, where the rotational speed must be controlled stepwise. Considering this reason, the SAE\#2 test setup used in this study is therefore modified without any flywheel.

Figure 1 shows the photograph and the schematic drawing of the SAE\#2 test setup used in this study. The hydraulic pump (8) provides enough oil flow from the oil tank (7) for the lubrication and actuation with the main pressure of 25 bar. The safety valve (10) protects the proportional valve (11), which is connected to the actuation line of the clutch (5). Before entering the clutch, the applied pressure is measured by the pressure sensor (12), and the signal is sent to the data acquisition system (17). The overload flow of the safety valve is used for the clutch lubrication. The outgoing flow of the oil from the clutch is fed back to the tank. The temperature of the outgoing oil flow is measured by a thermocouple (15), and the signal is sent to the data acquisition system. The clutch itself is on one side fixed to the frame through a torque measurement system (6), while the other side is connected, via a flexible coupling (4), to a DC-motor (2) which is controlled by an external drive (1). The shaft velocity of the motor is measured by an encoder (3), and the signal is sent to the data acquisition system.
2.2. Materials. As the torque capacity is limited by the design of the test setup, the clutch used in the study only contains one active friction disc, that is, two frictional contacts instead of ten frictional contacts recommended by the design. The lining material of the friction disc is paper-based type and commercially available. Moreover, the friction disc has a waffle groove pattern with the inner diameter $d_{i}$ of $99 \mathrm{~mm}$ and the outer diameter $d_{o}$ of $133 \mathrm{~mm}$. The commercial ATF used for the clutch has a kinematic viscosity of $56 \mathrm{~mm}^{2} / \mathrm{s}$ (cSt) and $9.3 \mathrm{~mm}^{2} / \mathrm{s}$ at $40^{\circ} \mathrm{C}$ and $100^{\circ} \mathrm{C}$, respectively.

2.3. Experimental Procedure. Figure 2 shows the flowchart describing the experiments carried out in the study. There are four consecutive tests to be carried out that are discussed in the subsequent paragraphs. Given a fresh clutch, the experiment is first started with the run-in test, then continued with the accelerated life test (ALT), the stationary Stribeck test, and finally with the dynamic Stribeck test. These test sequences are repeated until the clutch is considered to have failed. In this approach, all the test parameters are designed such that the clutch would fail in a reasonable period of time. It should be mentioned here that the stationary and dynamic Stribeck tests are carried out when the oil temperature is in the range of $80-90^{\circ} \mathrm{C}$.

2.3.1. Run-In Test. The run-in test designed for this study, in principle, mimics the engagement process of a clutch in automatic transmissions. While the clutch is in open condition (i.e., disengaged phase), the shaft velocity of the motor is controlled at a certain value. When this desired velocity is attained, a certain current signal profile is sent to the proportional valve such that a desired pressure profile, for example, see Figure 3, which is discussed in the following paragraphs, is built up to actuate the clutch. The run-in test is carried out for 200 engagement cycles.

The pressure profile is designed as follows. Initially, there is no electric current signal sent to the proportional valve, such that the clutch pressure is zero $\left(p_{1}=0\right)$ and consequently no torque is transmitted. In order to actuate the clutch, the oil flow to the clutch is suddenly increased causing the pressure to increase to the level of $p_{2}$, thus allowing the piston to move from its rest position. This pressure level is maintained for a certain duration and afterwards the flow is decreased. As a result, the pressure drops to the pressure level of $p_{3}$, which is intended to decelerate the piston motion and to allow the piston to standstill. This condition should be selected such that the discs do not make contact yet with each other. The previous sequence is referred to as the filling phase. In this particular phase, the pressure level $p_{3}$ is chosen such that the elastic force of the returning spring is overcome, but the piston should not move at high velocity with respect to the discs. Since the plates do not make contact yet, the torque that is already being transferred is only due to the viscous effect. The phase following the filling phase is referred to as the engagement phase, where the pressure is gradually increased linearly $\left(p_{4}\right)$ until the maximum pressure is reached, so that more and more discs are pressed together. In this study, the maximum pressure is set to 8 bar. 


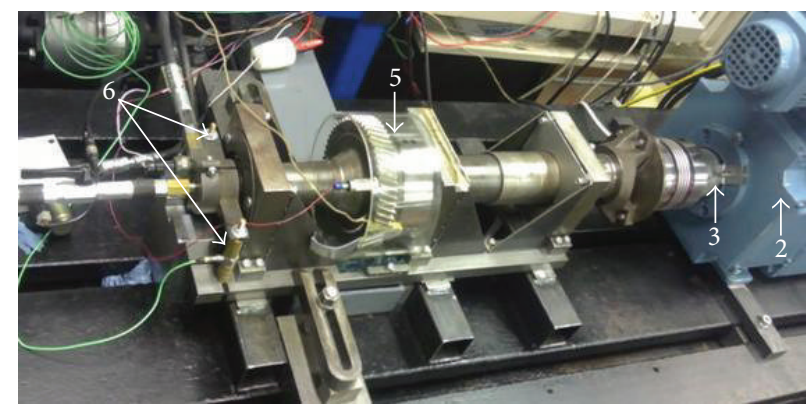

(a)

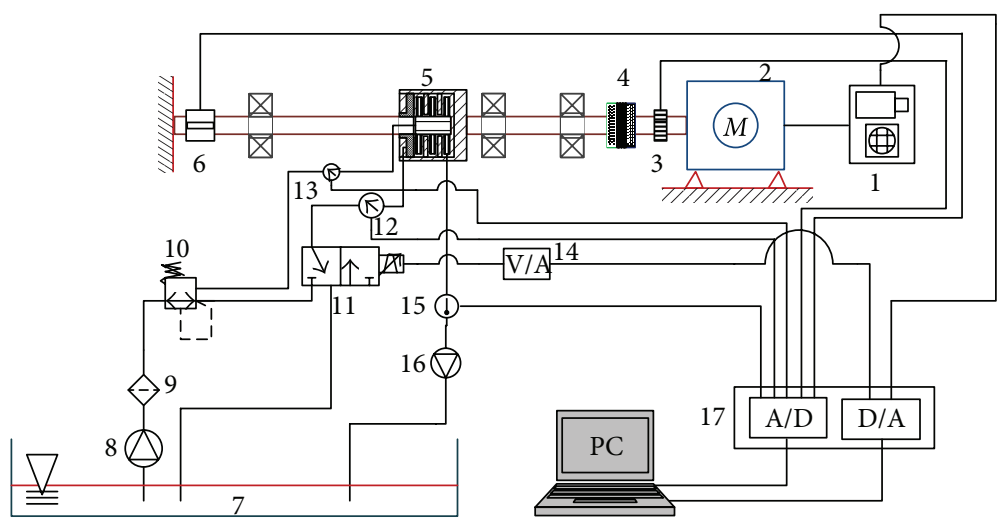

(b)

FIGURE 1: Modified SAE\#2 test setup without a flywheel.

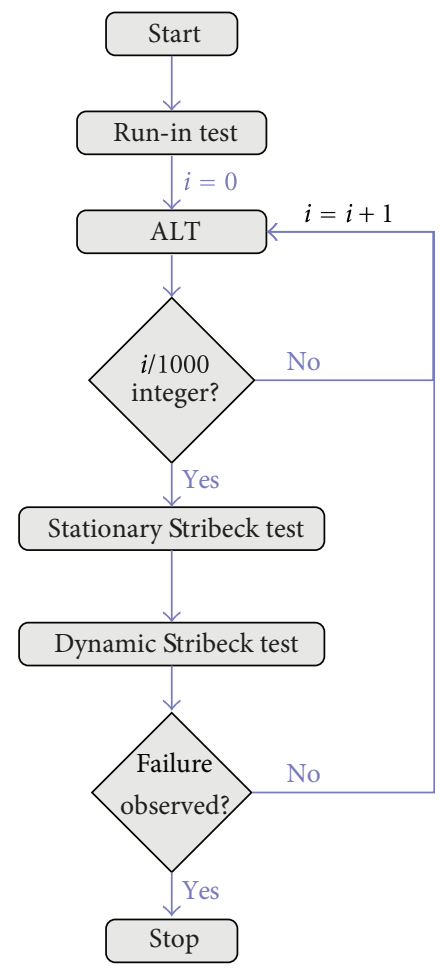

FIGURE 2: A flowchart describing the test sequences.
The control mode of the motor is a velocity control in which the maximum torque of the motor is limited to a certain value. In this study, the velocity is set to $1200 \mathrm{rpm}$ and the maximum torque is set to $20 \mathrm{Nm}$ during the test. As the applied pressure increases, the resulting friction torque increases and the motor delivers more torque to maintain the desired velocity. However, since the maximum torque of the motor is limited, the motor will decelerate when the resulting friction torque exceeds the limit torque and eventually will come to a standstill. The procedure proposed in this paper differs from the standard procedure in an SAE\#2 test setup as discussed in [4], where the electric motor is switched off during the engagement. Here, the standard procedure is not possible to implement because of the absence of a flywheel on the test setup. As the inertia is very low on the test setup, the input shaft will directly stop when the motor is switched off. An additional advantage of the motor torque being kept constant during the test is that there is more energy dissipated in the clutch, so that the lifetime of the clutch could be reached in a shorter time.

2.3.2. Accelerated Life Test (ALT). In principle, the accelerated life test (ALT) is carried out in the same manner as the run-in test discussed previously. However, the energy applied during the ALT is higher than that in the run-in test. For this purpose, the shaft velocity of the motor is set to $2000 \mathrm{rpm}$. 


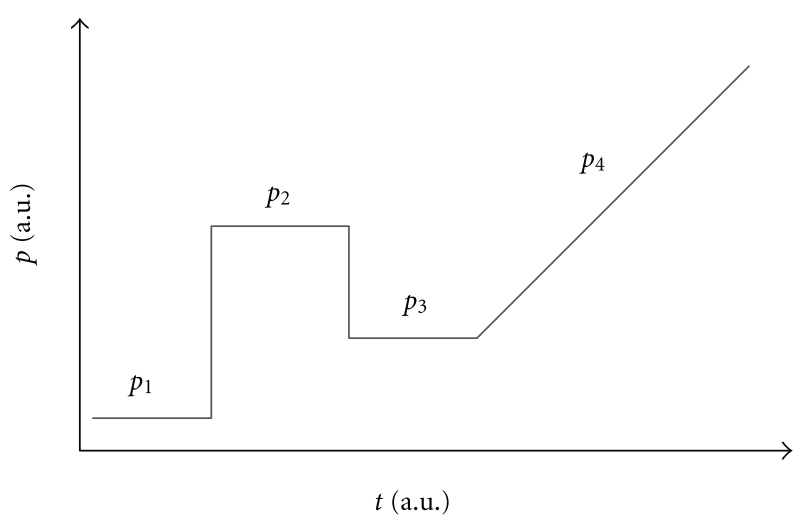

FIGURE 3: A typical pressure profile applied in the run-in test and ALT.

2.3.3. Stationary Stribeck Test. The main objective of the stationary Stribeck test is to determine the stationary Stribeck curve as a function of the clutch lifetime. It is important to note here that the stationary Stribeck curve is derived from a set of friction torques measured at constant relative velocity and constant applied pressure. In this study, the stationary Stribeck curve is determined for the applied pressure ranging from 4 to 7 bar and for the relative velocity ranging from 20 to $1970 \mathrm{rpm}$. The pressure step is 0.5 bar and the velocity step is $50 \mathrm{rpm}$.

For a given pressure level, the velocity is increased stepwise from 20 to $1970 \mathrm{rpm}$, where the duration for a given velocity level is set to $5 \mathrm{~s}$. After the friction torques for the entire velocity range have been measured, the pressure is increased by 0.5 bar, and in the new pressure level the velocity is restarted from 20 to $1970 \mathrm{rpm}$. This procedure is repeated until the maximum pressure level, that is, 7 bar, is attained.

2.3.4. Dynamic Stribeck Test. The dynamic Stribeck test aims at determining the dynamic frictional behavior of the clutch in nonstationary conditions as a function of the clutch lifetime. As mentioned previously, the dynamic frictional behavior is typified by the frictional lag phenomenon. When the relative motion is accelerated the resulting friction torque is higher than the friction torque in stationary condition. In contrast, when the relative motion is decelerated, the resulting friction torque is lower than the stationary friction torque. For a sinusoidal relative motion where acceleration and deceleration are evident, a loop is observable around the stationary Stribeck curve. The loop area is strongly determined by the frequency of the motion. In the GMS friction modeling framework $[5,6]$, the frictional lag phenomenon is determined by the attractor parameter $C$. The higher the attractor parameter is, the faster the resulting friction torque will follow the stationary Stribeck curve, that is, smaller loop area.

The dynamic Stribeck test is carried out at different constant pressures ranging from 4 to 7 bar. For a given pressure level, a sinusoidal velocity profile having frequency of $0.1 \mathrm{~Hz}$, amplitude, and offset of $1000 \mathrm{rpm}$, respectively, is imposed. The total duration of the dynamic Stribeck test for a given velocity profile is $15 \mathrm{~s}$.

\section{Results and Discussion}

This section discusses the results of the stationary and dynamic Stribeck tests. From the tests, the friction torque as a function of three variables, namely, (i) velocity, (ii) pressure, and (iii) degradation level (i.e., number of duty cycles) are obtained. The experimental results of the stationary Stribeck tests are first discussed. Afterwards, the Stribeck parameters identified at different degradation levels and their evolutions during the clutch lifetime are discussed. After addressing the stationary Stribeck characteristics and the evolution of the Stribeck parameters, the dynamic Stribeck behavior, which is characterized by the attractor parameter, is then discussed. Furthermore, the characteristics of the attractor parameter identified at different pressures, frequency excitations, and degradation levels are addressed.

For comparison purposes, photos, and surface profiles of the active friction disc are taken from the same wafle block (see Figure 4) prior and after the test. The photos are captured using an optical microscope and the surface profiles are measured along the sliding direction using a talysurf profilometer. At the same wafle block, the surface profiles are measured on eight different radial locations before and after the tests. The representative surface profiles of the wafle block before and after the tests are depicted in Figure 5. The average surface roughness $R_{a}$ from the surface profile measurements before the tests is $9.52 \mu \mathrm{m}$ and after the tests it is $1.94 \mu \mathrm{m}$. The average skewness $R_{\text {sk }}$ before the test is -0.87 and after the test it is -3.4 .

Figures 4 and 5 clearly show that the friction material has become smooth and flat after the tests, which indicates that the friction material has degraded during the tests. One can also notice in Figure 4 that the surface of the friction material before the tests is more porous compared to after the tests. The reduction of the surface porosity is believed to be caused by the pores blocking $[7,8]$, resulting from the deposition of debris particles of the friction material and/or degradation products of the ATF.

\subsection{Stationary Stribeck}

3.1.1. Experimental Results. Figure 6 shows the stationary Stribeck curve measured after the run-in test (i.e., initial condition), which is plotted both in $2 \mathrm{D}$ and $3 \mathrm{D}$. From the 2D Stribeck curve shown in Figure 6(a), the viscous effect dominates the friction torque at lower pressure (i.e., 4 bar), where the friction torque slightly increases with the velocity. At higher pressure, a different behavior can be observed in the figure, where the friction torque increases at lower velocity (positive slope) and decreases for higher velocity (i.e., Stribeck effect). The positive slope exhibited at lower velocity indicates that the clutch system has an anti-shudder property, which is possibly activated by the presence of friction modifier additive in the used ATF. In addition, it is also clear from the figure that the slope of the curves at lower velocity becomes more positive for higher applied pressure implying that the antishudder property becomes more pronounced at higher pressure. 


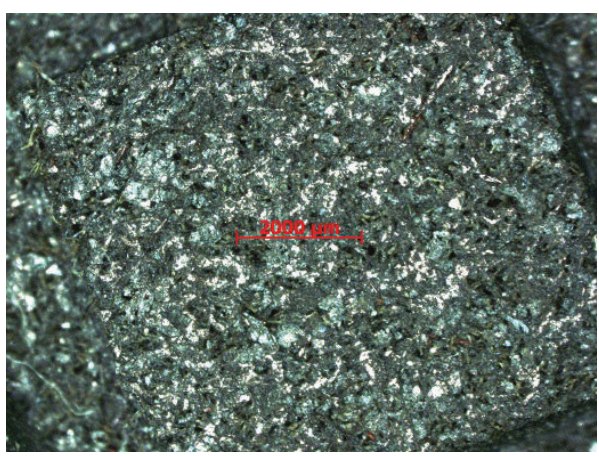

(a)

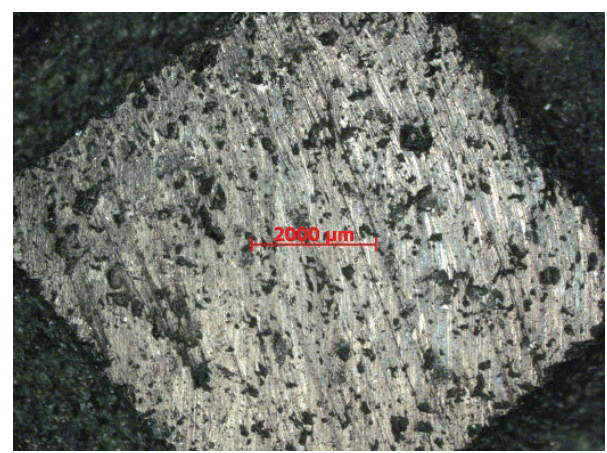

(b)

FIGURE 4: Photographs of the friction disc (a) before and (b) after the tests.

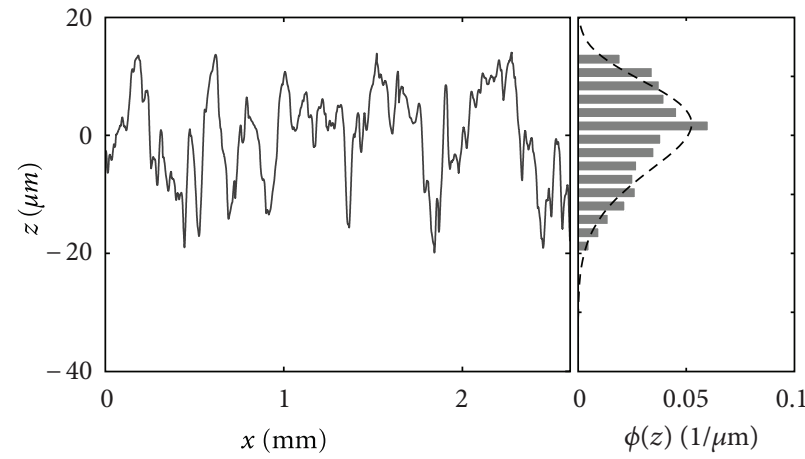

(a)

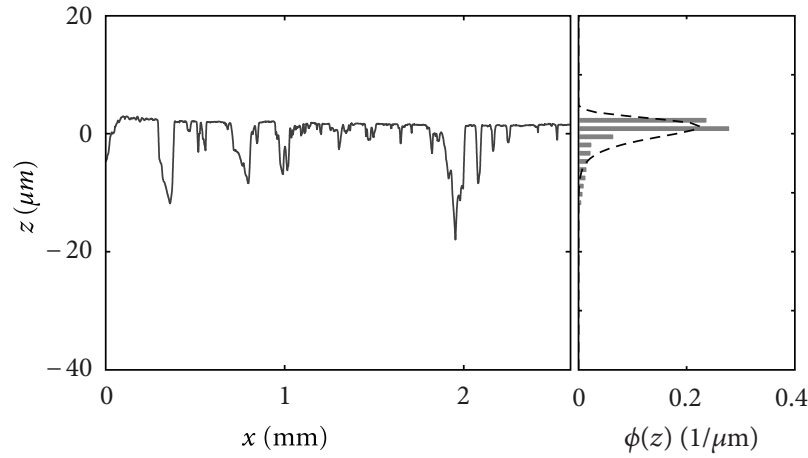

(b)

FIGURE 5: Surface profiles and the distributions $\phi(z)$ of the friction disc (a) before and (b) after the tests.

The evolution of the Stribeck curve with the progression of degradation is shown in Figure 7. Figure 7(a) shows the evolution of the Stribeck curve for all pressures, and Figure 7(b) shows the evolution of the Stribeck curve at 5 bar. The Stribeck curves represent the measurements obtained from the initial state, after 1000, 7000, and 15000 duty cycles of ALT. The condition after 15000 duty cycles is considered to be fully degraded state, where the mean coefficient of friction (COF) has been reduced to $50 \%$ from the initial mean $\mathrm{COF}$ [9].

It is evident in Figure 7 that the overall friction torque decreases with the progression of the clutch degradation. A drastic decrease of the friction torque is observed in the beginning of the clutch lifetime and the friction torque tends to saturate when the clutch degradation proceeds further. This implies that the overall (i.e., averaged) clutch coefficient of friction (COF) decreases with the progression of the clutch degradation, which is consistent with the experimental observations reported in the literature $[4,10]$. In many works of literature, the decrease of the COF is widely accepted to be caused by the loss of the surface porosity of the friction material (see Figure 4). Low surface porosity implies that the ATF is not easily evacuated from the contacting surfaces, so that the contact between the friction and separator discs is predominantly controlled by the ATF. Nevertheless, the thermal degradation occurring in the friction material can also contribute to the reduction of the COF [11].

One can also notice the effect of the clutch degradation on the slope of the Stribeck curve in Figure 7(b). The positive slope at lower velocity, that is, the antishudder property, is lost due to the clutch degradation and the slope becomes more negative at lower velocity with the progression of the clutch degradation.

3.1.2. Identification Results. In order to characterize the evolution of the stationary Stribeck curve, the parameters governing the curve, that is, the Stribeck parameters, are identified at different degradation levels. In the following analysis, the rotational velocity in $\operatorname{rpm} n_{\omega}$, which is obtained from the tests, is converted to the sliding velocity $v$ by the following equation:

$$
v=\frac{2 \pi n_{\omega} r_{m}}{60}
$$

with $r_{m}=\left(d_{i}+d_{o}\right) / 2$ denoting the mean radius of the friction disc. At a given state (i.e. degradation level), the Stribeck 


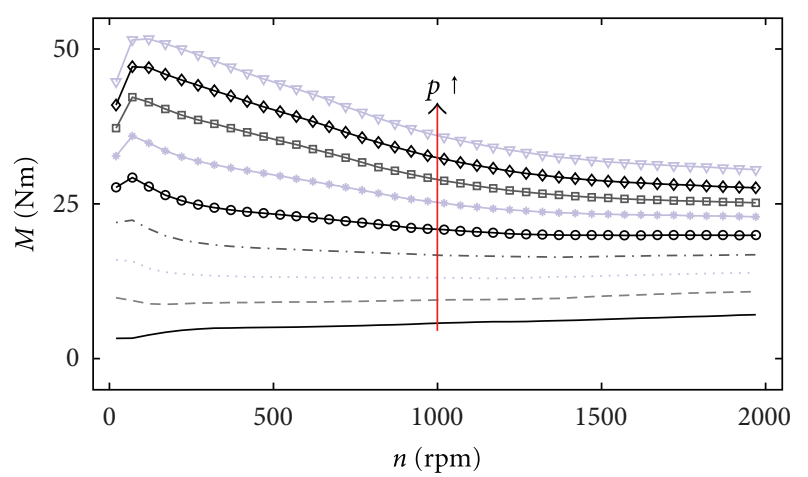

(a)

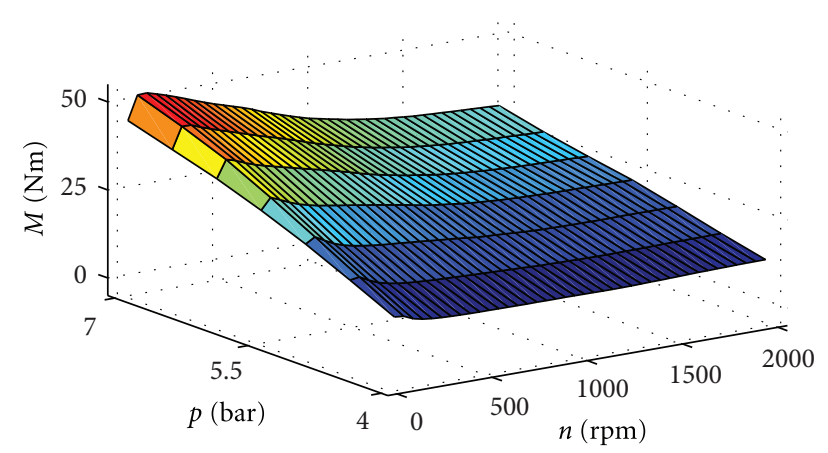

(b)

FIGURE 6: The stationary Stribeck curve measured at initial condition. (a) The 2D Stribeck curve and (b) the 3D Stribeck curve.

curve as a function of pressure $p$ and sliding velocity $v$ can be modeled according to the following equation [12]:

$$
\begin{gathered}
S(v, p)=\operatorname{sign}(v)\left[M_{s}(p)\left(1+\left[\bar{M}_{c}(p)-1\right] e^{-\left|V_{s}(p) / v\right|}\right)\right. \\
\left.+C_{v}(p)|v|^{\gamma}\right]
\end{gathered}
$$

where $M_{s}(p)$ is the static friction torque, $\bar{M}_{c}(p)$ is the ratio between the Coulomb friction torque and the static friction torque, $V_{S}(p)$ is the Stribeck velocity, and $C_{v}(p)$ is the viscous constant at pressure $p$.

In [12], it has been shown that the typical value of the parameter $\gamma$ is 0.3 . By keeping this parameter constant, the complexity of the model in (2) is reduced from five Stribeck parameters to four Stribeck parameters. Hence, the shape of the Stribeck curve is now determined by the four parameters, $M_{s}(p), \bar{M}_{c}(p), V_{S}(p)$, and $C_{v}(p)$, which are pressure dependent. These pressure dependencies are determined from the experimental data, as will be shown later.

Since the function expressed in (2) is highly nonlinear and in order to guarantee that the parameters to be identified have physical meaning, that is, the Stribeck parameters must be positive, the parameter identification thus constitutes a constrained nonlinear optimization problem. Here, the identification is performed separately for different pressures

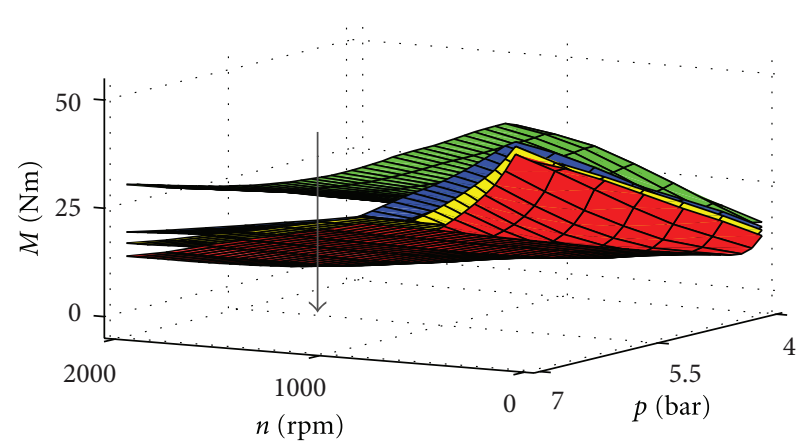

(a)

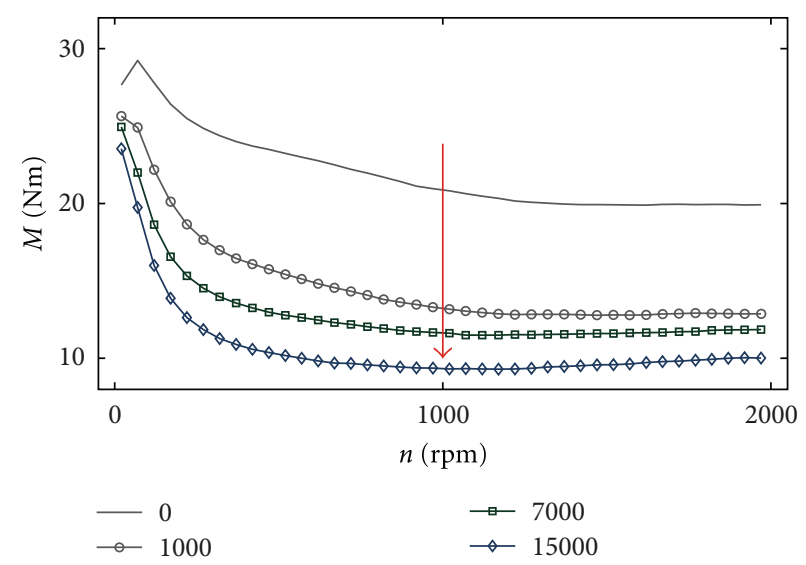

(b)

FIGURE 7: The stationary Stribeck curves measured for different degradation levels. (a) the evolution of the 3D Stribeck curve and (b) The evolution of the $2 \mathrm{D}$ Stribeck curve at 5 bar. Note that the arrow indicates the progression of degradation.

and states (i.e., degradation level). At a certain pressure and state, the Stribeck curve is therefore expressible as

$$
S(v)=\operatorname{sign}(v)\left[M_{s}\left(1+\left[\bar{M}_{c}-1\right] e^{-\left|V_{S} / v\right|}\right)+C_{v}|v|^{0.3}\right] .
$$

Thus, the optimization problem can now be formulated as

$$
\begin{aligned}
& \underset{\mathbf{S}}{\operatorname{minimize}} f_{0}(\mathbf{S}), \\
& \text { subject to } \mathbf{S} \geq 0 \text {, }
\end{aligned}
$$

where $f_{0}(\mathbf{S})$ represents the objective function, that is, the percentage mean-square-error (MSE), which is defined by

$$
f_{0}(\mathbf{S})=\frac{1}{N \sigma_{M_{\exp }}^{2}} \sum_{i=1}^{N}\left[S\left(v^{i}\right)-M_{\exp }^{i}\right]^{2} \times 100[\%],
$$

with $\mathbf{S}=\left[M_{s}, \bar{M}_{c}, V_{S}, C_{v}\right]$ denoting a vector containing the Stribeck parameters at a certain state and pressure, $M_{\exp }^{i}$ denoting the measured torque at sliding velocity $v^{i}, \sigma_{M_{\text {exp }}}^{2}$ denoting the variance of $M_{\exp }^{i}$, and $N$ denoting the number 


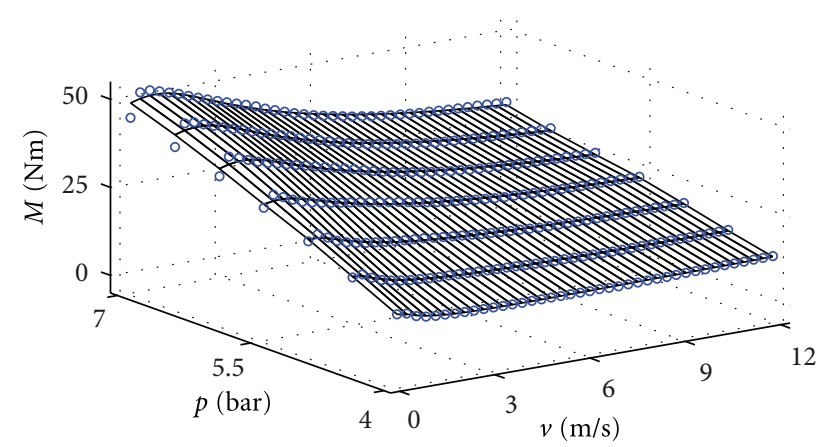

FIgURE 8: Comparison between the Stribeck curve measured at initial condition and the model.

of the velocity steps. The MSE can be viewed as the goodness of fit between the experimental data and the model, where, as a rule of thumb, the fitness is good when the MSE is smaller than $5 \%$.

For the Stribeck parameters identification, the interiorpoint algorithm $[13,14]$ is used to solve the optimization problem formulated in (5). Figure 8 shows the comparison between the 3D Stribeck curve (the circle markers), measured at initial condition, and the modeled curve (surface) obtained based on the aforementioned identification procedure. The average MSE obtained from the identification of the Stribeck curve measured at initial condition for different pressures is $1.74 \%$ and the maximum MSE is $3.61 \%$.

Figure 9 shows the pressure dependence of the Stribeck parameters. The circle markers denote the identified values for different pressures and the solid lines denote the trends. It can be seen that the three Stribeck parameters $\left(M_{s}, V_{S}\right.$, and $C_{v}$ ) increase linearly with the applied pressure $p$, while the parameter $\bar{M}_{c}$ decreases asymptotically with the applied pressure $p$. The pressure-dependent relationships of the Stribeck parameters are based on the formulations discussed in [12].

Figure 10 shows the evolution of the Stribeck parameters identified at a pressure of 5 bar (circle markers) in function of the ALT duty cycles and the general trend (the solid lines) estimated using the degradation model expressed as [15]

$$
\xi=\xi_{0}+\Delta\left[1-\left(\frac{1}{1+\zeta / \varphi}\right)^{\epsilon}\right]
$$

with $\xi$ denoting a Stribeck parameter at an arbitrary state, $\xi_{0}$ denoting the Stribeck parameter at an initial state, $\Delta, \varphi$, and $\epsilon$ being the degradation model coefficients that, respectively denote the maximum deviation from $\xi_{0}$, the scaling factor of the abscissa, and the characteristic exponent, and $\zeta$ denoting a variable that represents the progress of degradation, for example, number of cycles or sliding distance.

For this particular pressure, one can see that the three parameters, namely $M_{s}, \bar{M}_{c}$, and $V_{S}$, tend to decrease with the progression of clutch degradation. In contrast, the viscous parameter $C_{v}$ tends to increase with the progression of clutch degradation. The increasing trend of $C_{v}$ implies that the viscous effect becomes more dominant as the clutch degradation progresses. This tendency is consistent with the visual observation on the surface of the friction disc after the tests (see Figure 4), where the contact surface has lost its porosity, so that, as mentioned before, the resulting friction torque is predominantly controlled by the ATF property.

Furthermore, Figure 11 shows the overall evolutions of the identified parameters (circle markers) and the trends (surfaces) at different pressures. One can clearly see in the figure that the Stribeck parameters have similar trends in function of the number of duty cycles when observed at different pressures.

3.2. Dynamic Stribeck Tests. As mentioned previously, the dynamic Stribeck test in this study is carried out by applying an imposed sinusoidal velocity profile with a frequency of $0.1 \mathrm{~Hz}$, where the pressure is kept constant during the velocity excitation. In this way, the effects of acceleration and deceleration on the resulting friction torque can be observed. Due to acceleration and deceleration, the friction deviates with respect to the steady-state behavior, that is, the stationary Stribeck curve, thus resulting in the formation of a hysteresis loop which is located around the stationary Stribeck curve, that is referred to as the frictional lag effect.

Figure 12 shows the dynamic Stribeck curve measured at 5 bar, where the effects of acceleration and deceleration on the resulting friction are visible. It can be seen in the figure that the resulting friction is higher during the acceleration than that during the deceleration. The physical explanation for this phenomenon is widely associated with the "squeeze film" effect [16]. As discussed in [16], the squeeze film force $F_{\text {sq }}$ on a lubricated line contact is given by

$$
F_{\mathrm{sq}}=-\eta_{e} l\left(\frac{2 a}{h_{c}}\right)^{3} \frac{\partial h_{c}}{\partial t}
$$

with $\eta_{e}$ denoting the effective dynamic viscosity, $l$ denoting the length of line contact, $a$ denoting the half width of Hertzian contact, and $h_{c}$ denoting the average film thickness. During the acceleration, the film thickness increases $\left(\partial h_{c} / \partial \uparrow \uparrow\right)$ implying that the squeeze force is negative. In other words, the squeeze force acts as an external load during the acceleration. As a result, the film thickness during acceleration is less than that during stationary condition. This suggests that the number of asperity-to-asperity contacts becomes higher in the acceleration with respect to the stationary condition, thus resulting higher friction. In contrast, the film thickness decreases during the deceleration phase, implying that the squeeze pressure becomes positive which contributes to the load-carrying capacity. This results in a higher film thickness compared with the corresponding value in the stationary lubrication, which translates to a lower friction. A measure that represents the frictional lag effect can be simply defined as the loop area $A_{\text {lag }}$ of the friction curve, which is expressible as

$$
A_{\text {lag }}=\oint M d v
$$

with $M$ and $v$, respectively, denoting the torque and the velocity. 


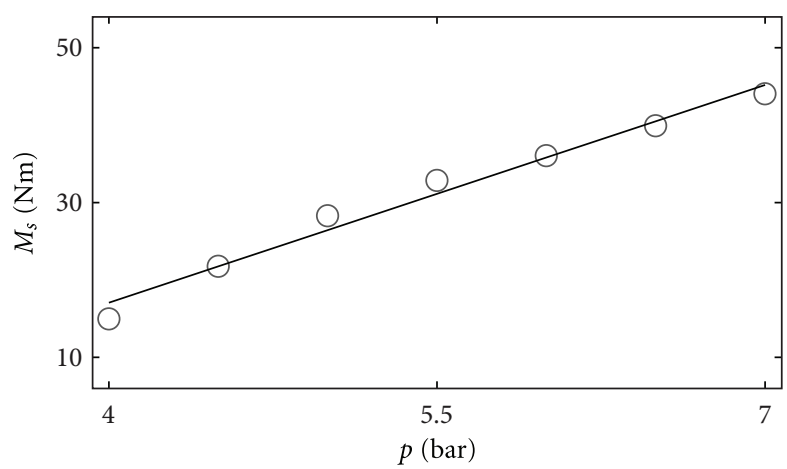

(a)

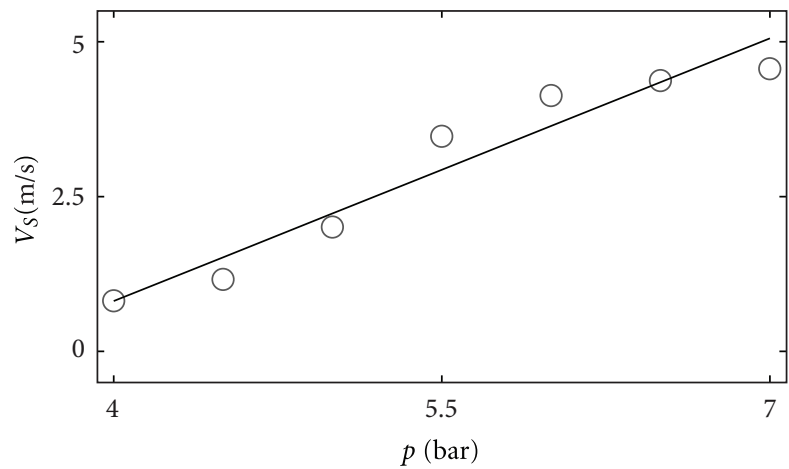

(c)

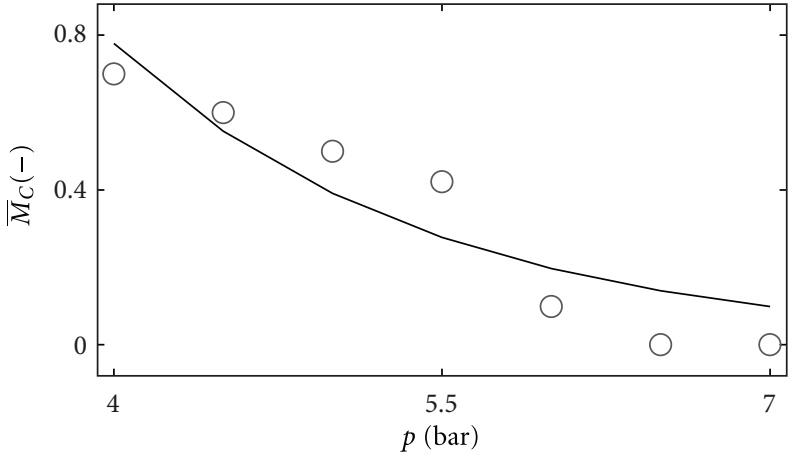

(b)

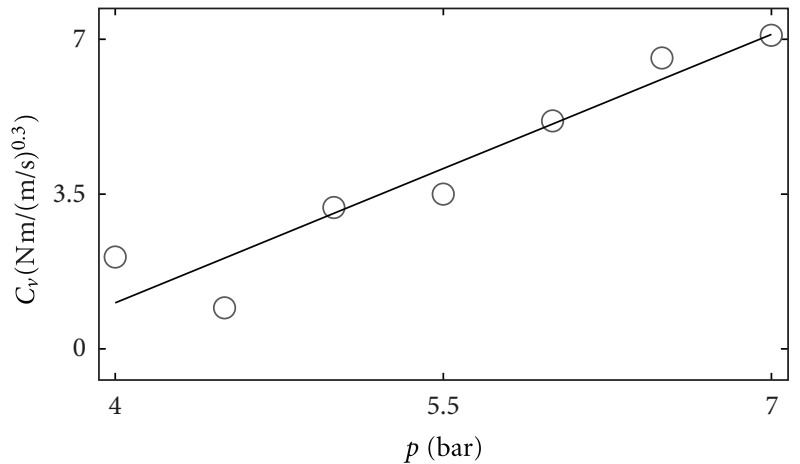

(d)

FIgURE 9: The identified Stribeck parameters at initial condition in function of applied pressure.

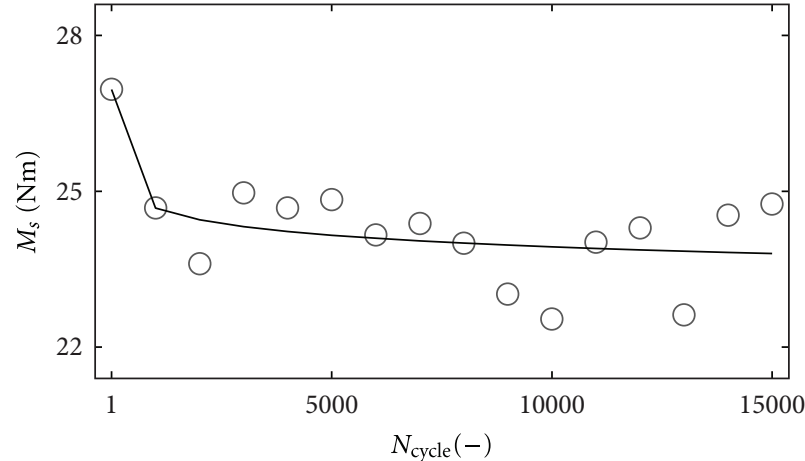

(a)

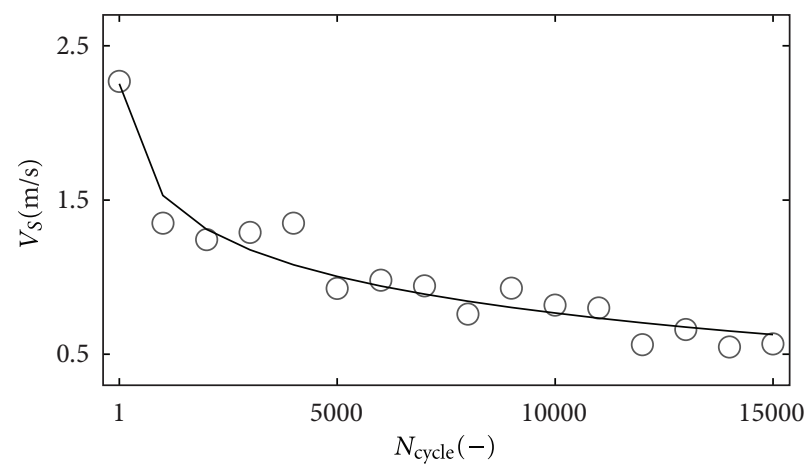

(c)

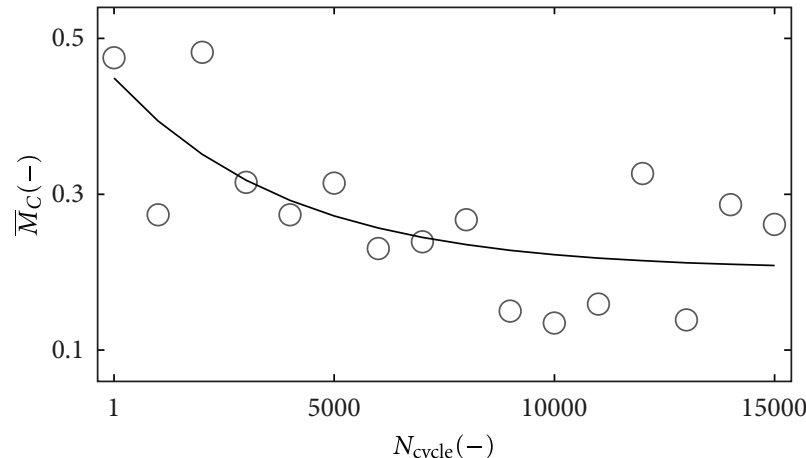

(b)

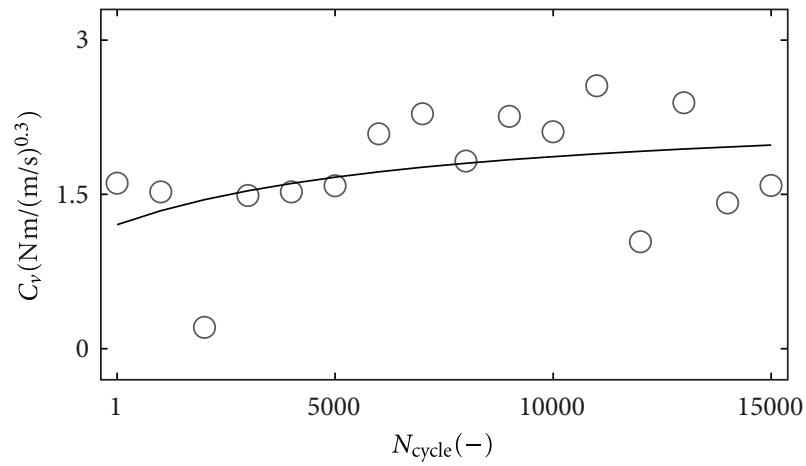

(d)

Figure 10: The evolution of the identified parameters of the Stribeck curve measured at applied pressure of 5 bar in function of the duty cycles. 


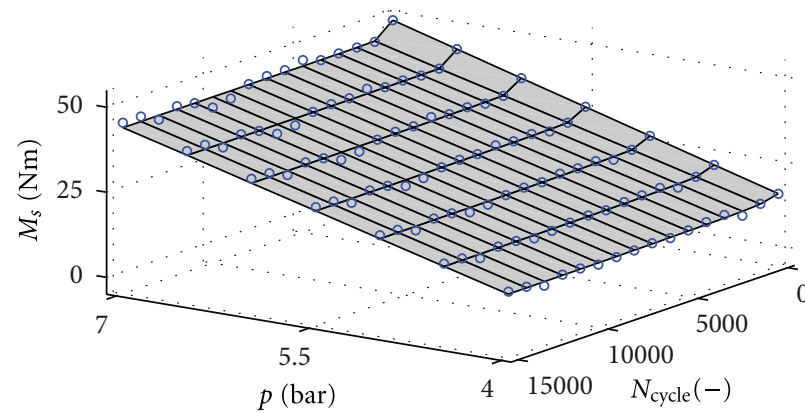

(a)

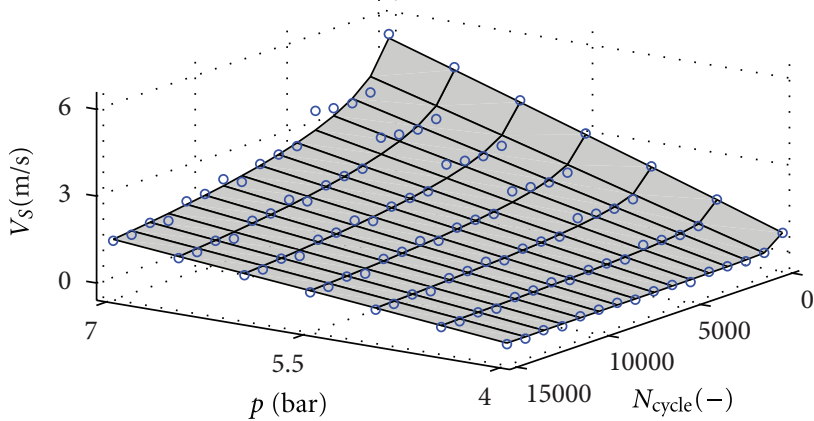

(c)

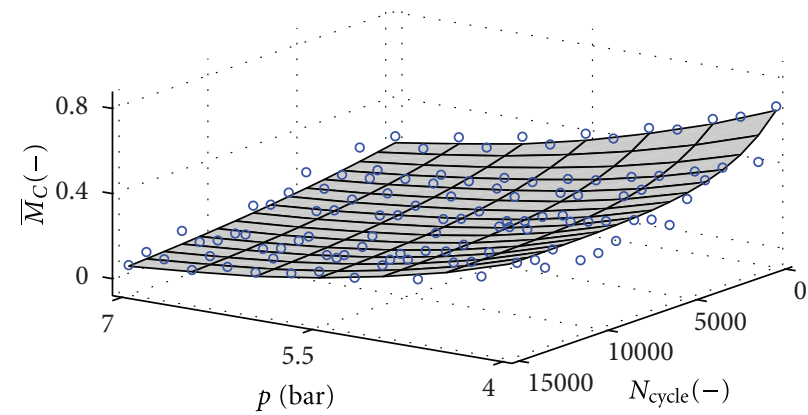

(b)

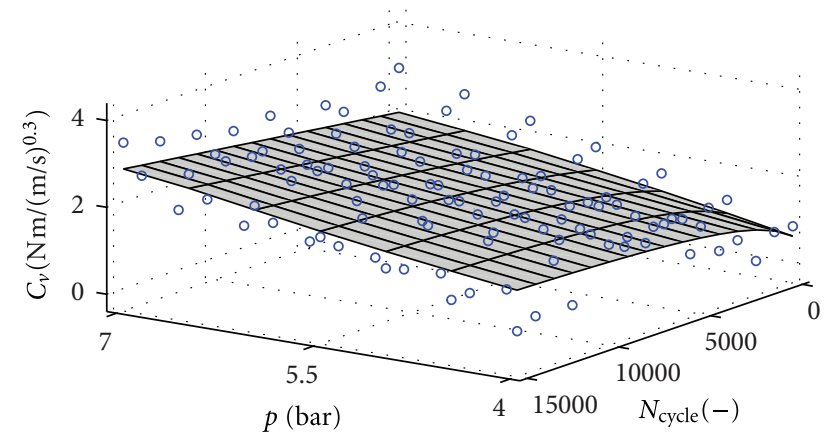

(d)

FIGURE 11: The overall evolutions of the identified parameters of the Stribeck curves measured at different pressures in function of the duty cycles.

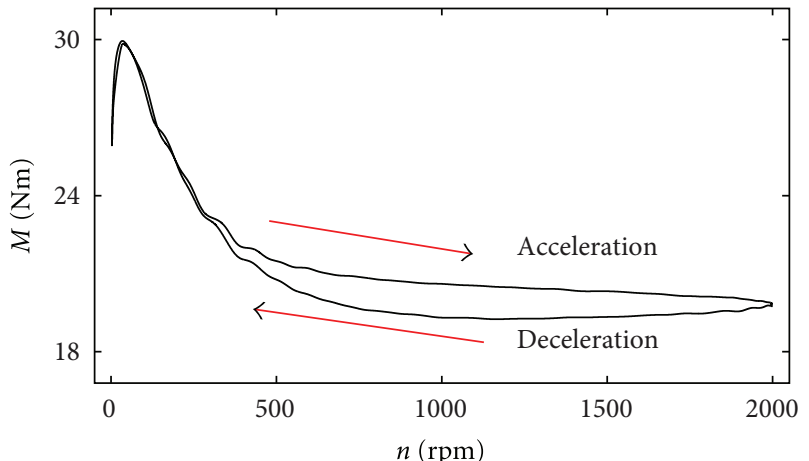

FIGURE 12: Dynamic Stribeck curve of the fresh clutch measured at 5 bar.

The effect of the applied pressure on the frictional lag phenomenon at initial condition (fresh clutch) is shown in Figure 13. Visually, one may notice that the loop area increases with an increase of applied pressure. This observation is qualitatively identical to the simulation results presented in [16]. As the pressure increases, the average film thickness decreases. As revealed by (7), the squeeze film force is inversely proportional to the average film thickness $h_{c}$. This suggests that the squeeze film effect is more pronounced at higher pressure. Hence, the loop area $A_{\text {lag }}$ becomes larger at higher pressure, which can be clearly seen in Figure 14. Moreover, the figure also depicts that the loop area increases quite proportionally to the applied pressure.

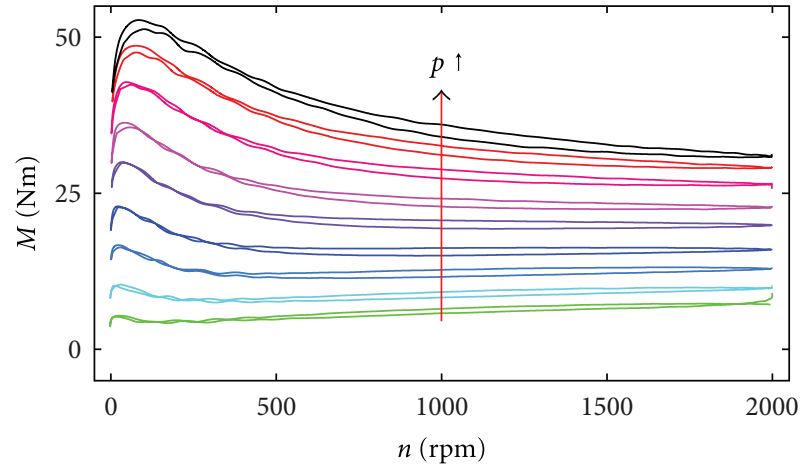

FIGURE 13: Dynamic Stribeck of the fresh clutch at different applied pressures.

Figure 15 shows the dynamic Stribeck measurements during the clutch lifetime at the applied pressure of 5 bar. As can be seen in the figure, the friction torque decreases with the progression of the clutch degradation, which is consistent with the stationary Stribeck measurements. Interestingly, one can see that the loop area increases during the clutch lifetime at this particular applied pressure, which is more visible in Figure 16. The possible physical explanation is as follows. It has been mentioned in the previous section that the friction material surface becomes smoother with the progression of clutch degradation (see Figure 5). For a rough surface, the asperities have a greater contribution in carrying the load which suggests that the squeeze effect does not play any 


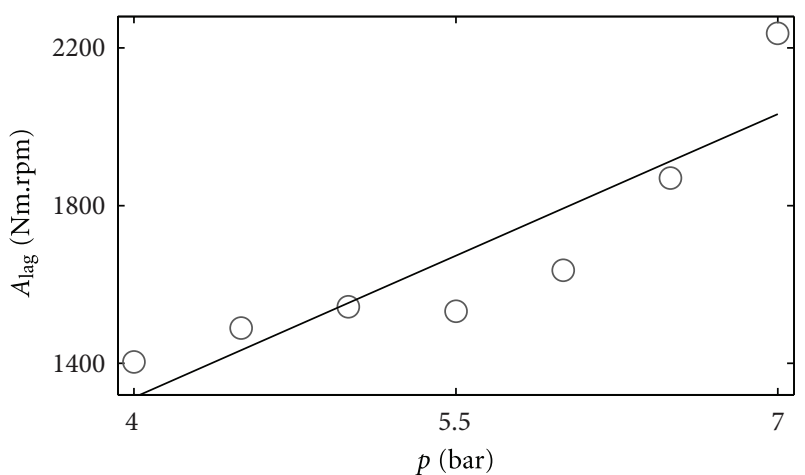

FIGURE 14: The loop area resulting from the frictional lag in function of applied pressure calculated based on the friction curves in Figure 13.

significant role in a rough surface if compared to a smooth surface [16].

It is now interesting to observe how the loop area $A_{\text {lag }}$ evolves during the clutch lifetime for different applied pressures. Surprisingly, the trends of $A_{\text {lag }}$ are exceptional at higher pressures. As can be seen in Figure 17, $A_{\text {lag }}$ increases with the progression of the clutch degradation at relatively lower pressure (4-6 bar). On the other hand, the evolution of $A_{\text {lag }}$ exhibits an opposite behavior at relatively higher pressures, where it tends to decrease with the progression of the clutch degradation. The possible physical explanation for this evidence is as follows. It is assumed that the surface porosity plays a significant role at higher pressure on the squeeze film due to the dynamic conditions. As the clutch degradation progresses, the friction material looses its surface porosity, namely, more surface pores are blocked (see Figure 4), thus resulting in the deterioration of the clutch capability to evacuate the oil from the contacting surfaces. This suggests that the average film thickness $h_{c}$ does not necessarily decrease for degraded friction material when the applied pressure is increased. In addition, the loss of surface porosity may also suggest that the rate of change of the film thickness $\partial h_{c} / \partial t$ at higher pressure becomes less pronounced in the presence of acceleration or deceleration.

\section{Conclusion}

The stationary Stribeck tests reveal that the stationary friction curve drops globally as the friction material degradation progresses. Four parameters, namely, (i) the static friction torque $M_{s}$, (ii) the ratio between the Coulomb friction torque and the static friction torque $\bar{M}_{c}$, (iii) the Stribeck velocity $V_{S}$, and (iv) the viscous effect $C_{v}$, are referred to as the Stribeck parameters that govern the stationary friction characteristics, that is, Stribeck curve, at a certain state. In order to characterize the change of the sliding friction characteristics, the four parameters need to be identified at different states. The identification results show that the Stribeck parameters appear to evolve deterministically during the clutch lifetime. The first three parameters $\left(M_{s}, \bar{M}_{c}\right.$, and $V_{S}$ ) tend to decrease with the progression of the degradation,

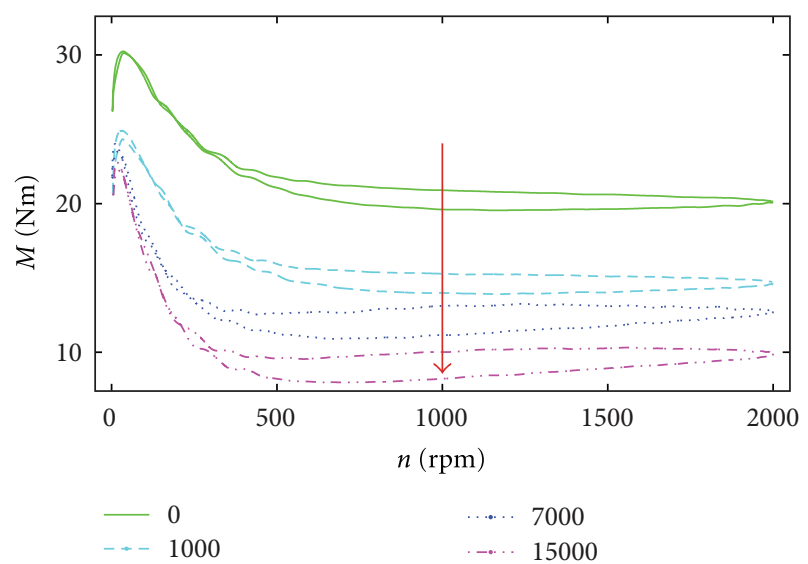

FIGURE 15: The evolution of the dynamic Stribeck curve at applied pressure of 5 bar.

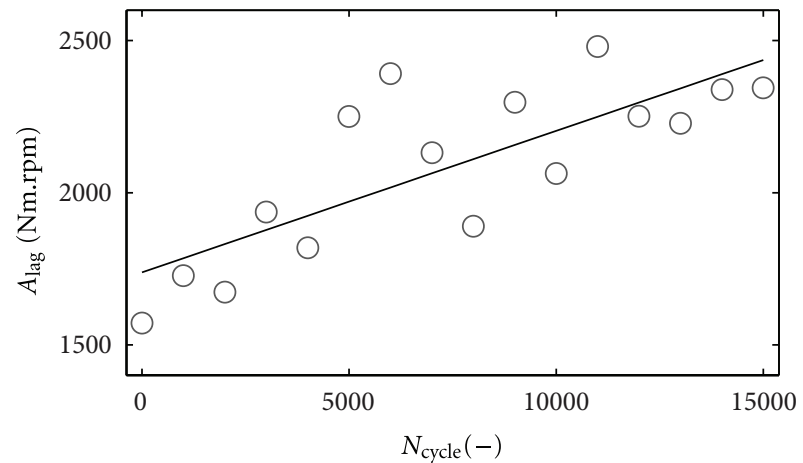

FIGURE 16: The evolution the frictional lag loop during the clutch service-life at applied pressure of 5 bar.

while the last parameter $\left(C_{v}\right)$ tends to increase. Increasing $C_{v}$ implies that the viscous effect becomes more pronounced as the clutch degradation progresses. This implication is confirmed by the visual observation on the surface of the fully degraded friction material. Based on the observation, it is evident that the degraded friction surface has lost its porosity. Thus, the ability of the degraded friction surface to evacuate the lubricant from the interface deteriorates, suggesting that the friction torque occurring in the clutch is predominantly controlled by the lubricant.

Under acceleration or deceleration, the resulting friction will deviate from the stationary friction (stationary Stribeck curve). To investigate this effect, the dynamic Stribeck tests have been carried out, wherein a sinusoidal velocity excitation is applied on the clutch. In this way, both acceleration and deceleration effects are present simultaneously in the measured friction, eventually creating a hysteresis loop around the stationary curve that is called the frictional lag effect. The loop area, which is simple to compute, can be considered as a measure of the frictional lag effect. Furthermore, it has been shown in this study that the applied pressure has also an effect on the loop area of the sliding hysteresis. In the beginning of clutch lifetime, the loop area increases with the applied pressure. However, this characteristic changes with 


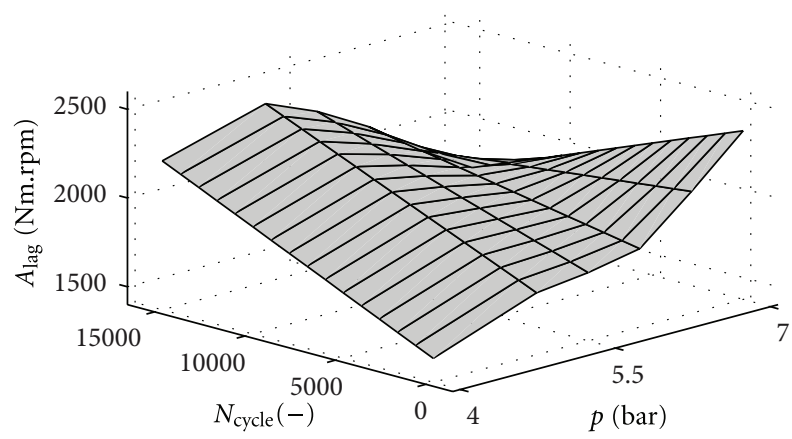

FIgURE 17: The evolution the frictional lag loop during the clutch service-life at different applied pressures.

the degradation progression, where the hysteresis loop area tends to decrease with the applied pressure at the end of the clutch lifetime.

\section{Acknowledgments}

All the authors would like to thank Tycho Van Peteghem and Wout Vandelaer for performing the experiments. Valuable comments of Professor Hendrik Van Brussel and Professor Farid Al-Bender on this study are appreciated. The experimental support from Dr. Mark Versteyhe of Dana-Spicer Off Highway Belgium is also acknowledged.

\section{References}

[1] F. Al-Bender, V. Lampaert, and J. Swevers, "A novel generic model at asperity level for dry friction force dynamics," Tribology Letters, vol. 16, no. 1-2, pp. 81-93, 2004.

[2] T. Tjahjowidodo, Characterization, modelling and control of mechanical systems comprising material and geometrical nonlinearities [Ph.D. thesis], Katholieke Unversiteit Leuven, Department of Mechanical Engineering, Division PMA, Leuven, Belgium, 2006.

[3] "SAE-International, SAE No. 2 Friction Test Machine Durability Test," Vol. SAE J2489, 2012.

[4] W. Ost, P. De Baets, and J. Degrieck, "The tribological behaviour of paper friction plates for wet clutch application investigated on SAE\#II and pin-on-disk test rigs," Wear, vol. 249, no. 5-6, pp. 361-371, 2001.

[5] V. Lampaert, F. Al-Bender, and J. Swevers, "A generalized maxwell-slip friction model appropriate for control purposes," in Proceedings of the IEEE International Conference on Physics and Control, 2003.

[6] F. Al-Bender, V. Lampaert, and J. Swevers, "The generalized Maxwell-slip model: a novel model for friction simulation and compensation," IEEE Transactions on Automatic Control, vol. 50, no. 11, pp. 1883-1887, 2005.

[7] S. Li, M. Devlin, S. H. Tersigni, T. C. Jao, K. Yatsunami, and T. M. Cameron, "Fundamentals of Anti-Shudder Durability: Part IClutch Plate Study," SAE Technical Paper 2003-01-1983, 51-62, 2003.

[8] T. Newcomb, M. Sparrow, and B. Ciupak, "Glaze Analysis of Friction Plates," SAE Technical Paper 2006-01-3244.
[9] T. VanPeteghem and W. Vandelaer, Levensduurvoorspelling van Transmissiesystemen voor Off-road Voertuigen [M.S. thesis], Katholieke Unversiteit Leuven, Department of Mechanical Engineering, Division PMA, Leuven, Belgium, 2011.

[10] J. Fei, H.-J. Li, L.-H. Qi, Y.-W. Fu, and X.-T. Li, "Carbon-fiber reinforced paper-based friction material: study on friction stability as a function of operating variables," Journal of Tribology, vol. 130, no. 4, Article ID 041605, 2008.

[11] A. P. Ompusunggu, P. Sas, and H. VanBrussel, "Influence of adhesive wear and thermal degradation on the frictional characteristics of paperbased friction materials: a comparative study," ISRN Tribology, vol. 2013, Article ID 739202, 2013.

[12] T. Janssens, Dynamic characterisation and modelling of dry and boundary lubricated friction for stabilisation and control purposes [Ph.D. thesis], Katholieke Unversiteit Leuven, Department of Mechanical Engineering, Division PMA, Leuven, Belgium, 2010.

[13] R. H. Byrd, J. C. Gilbert, and J. Nocedal, "A trust region method based on interior point techniques for nonlinear programming," Mathematical Programming B, vol. 89, no. 1, pp. 149-185, 2000.

[14] R. H. Byrd, M. E. Hribar, and J. Nocedal, "An interior point algorithm for large-scale nonlinear programming," SIAM Journal on Optimization, vol. 9, no. 4, pp. 877-900, 1999.

[15] A. P. Ompusunggu, Intelligent monitoring and prognostics of automotive clutches [Ph.D. thesis], Katholieke Unversiteit Leuven, Department of Mechanical Engineering, Division PMA, Leuven, Belgium, 2012.

[16] H. Sojoudi and M. M. Khonsari, "On the modeling of quasisteady and unsteady dynamic friction in sliding lubricated line contact," Journal of Tribology, vol. 132, no. 1, Article ID 012101, 9 pages, 2010. 

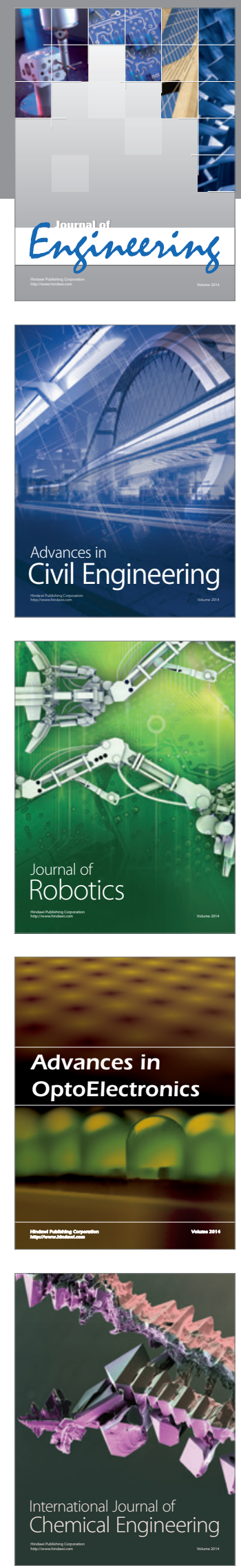

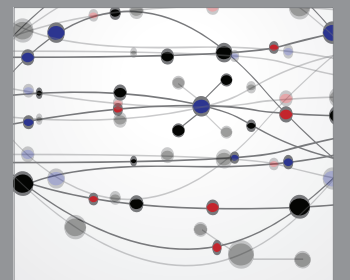

The Scientific World Journal
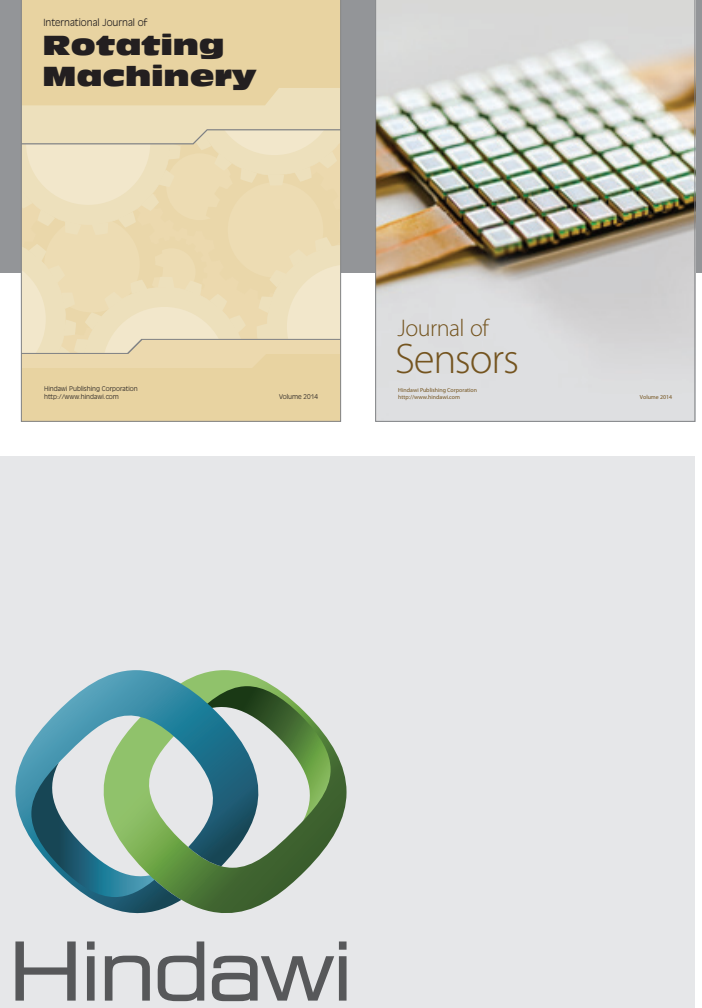

Submit your manuscripts at http://www.hindawi.com
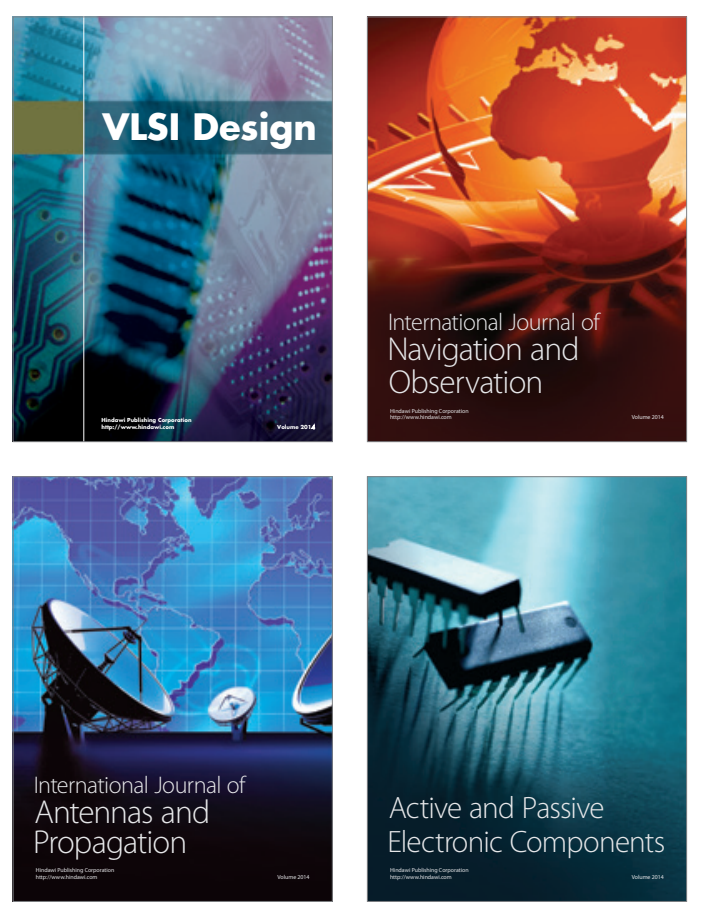
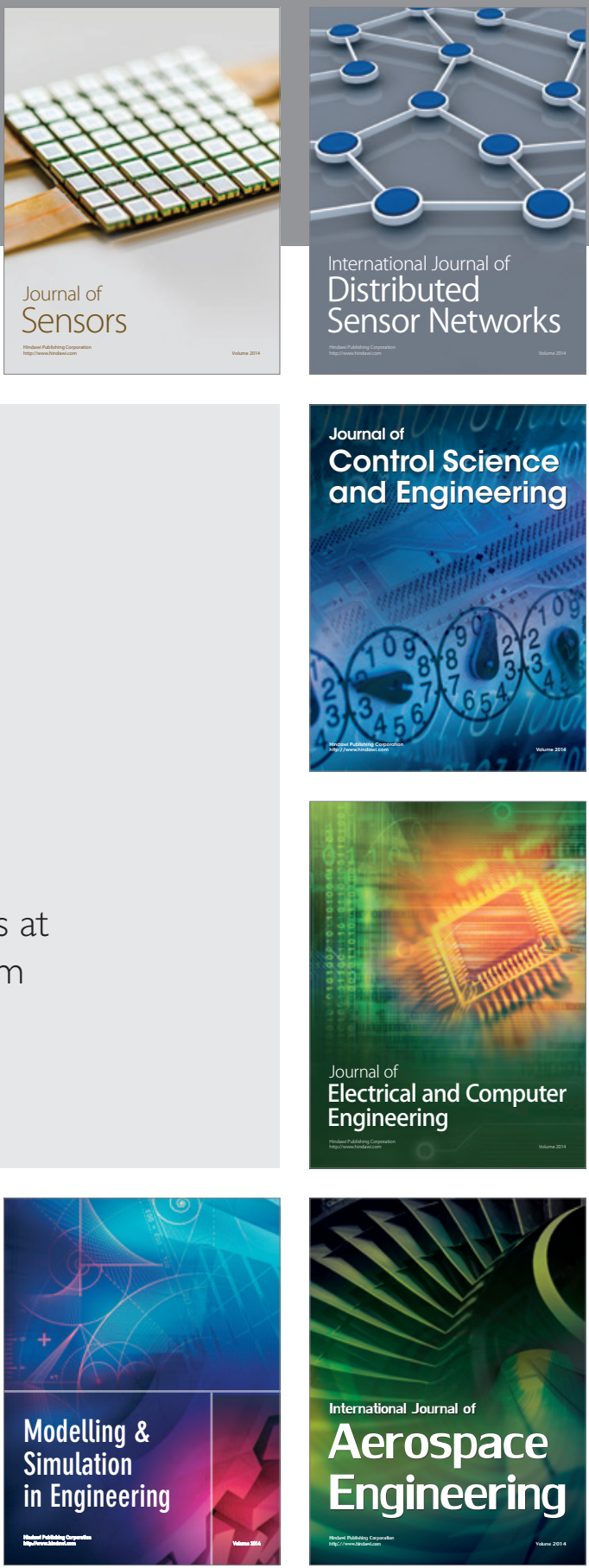

Journal of

Control Science

and Engineering
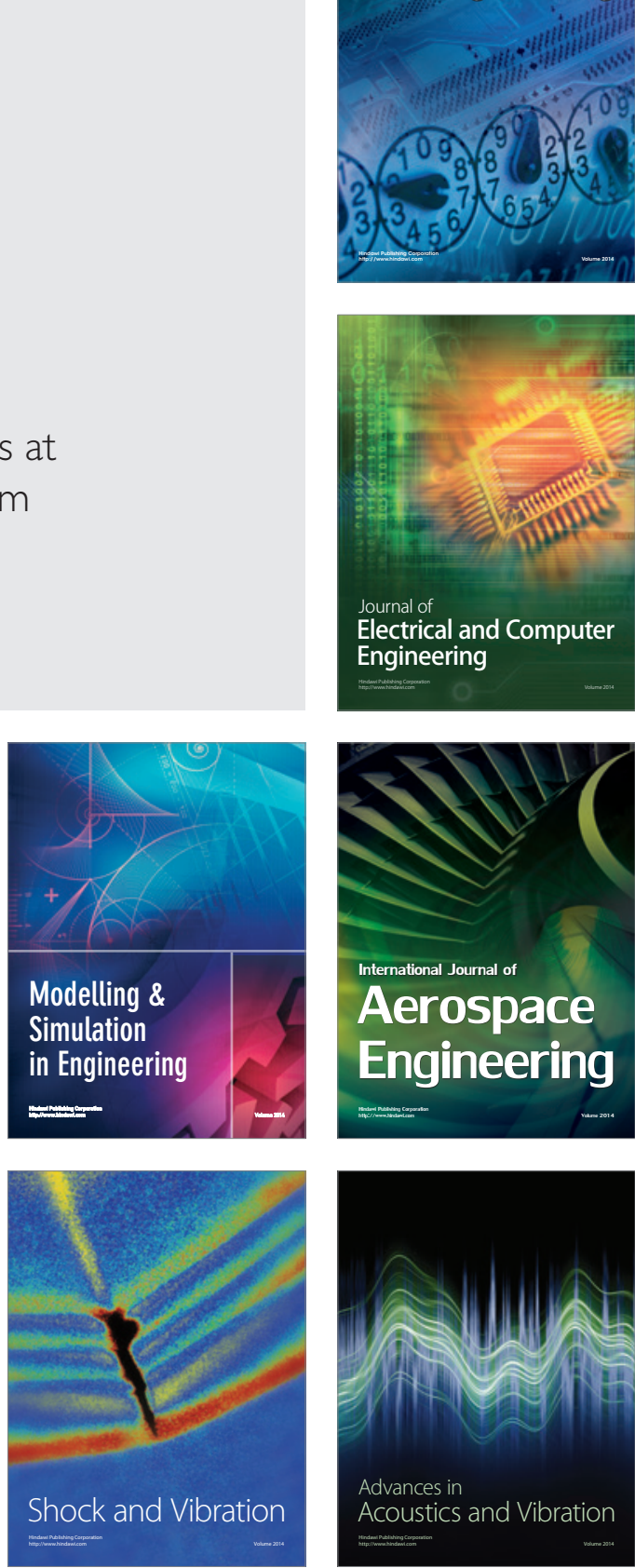